\title{
Prediction of the changes in drug dissolution from an immediate-release tablet containing two active pharmaceutical ingredients using an accelerated stability assessment program (ASAPprime ${ }^{\oplus}$ )
}

Hanlin $\mathrm{Li}^{1 *} \mathbb{D}$, David Nadig ${ }^{1}$, Andrew Kuzmission ${ }^{1}$ and Christopher M. Riley ${ }^{2}$

\begin{abstract}
The computer program ASAPprime ${ }^{\circledast}$ has been used successfully for some time to predict the stability of active pharmaceutical ingredients (APIs) in solid-dosage forms. In this study, we have demonstrated that the ASAPprime ${ }^{\circledast}$ program can also be used to predict the slow-down in dissolution of two APIs in an immediate release tablet. The tablets were pre-equilibrated at $25^{\circ} \mathrm{C}$ at different relative humidities (30-75\%), sealed in aluminum pouches and stored at temperatures ranging from $50-60{ }^{\circ} \mathrm{C}$ for 3,7 or 14 days. The storage times were selected to encompass the time needed to produce a slowdown in dissolution such that the amount of the two APIs fell below the acceptance criteria of no less than $80 \%$ dissolved in $20 \mathrm{~min}$. Up to 6 months of stability data from a $40^{\circ} \mathrm{C} / 75 \% \mathrm{RH}$ open dish study were also included in the modeling. The effects of temperature $\left(T\right.$ in $\left.{ }^{\circ} \mathrm{K}\right)$ and relative humidity $(\mathrm{RH})$ were then shown to be related to the isoconversion (IC) time by an empirical, modified Arrhenius equations, where IC is the time for the amount dissolved to equal $80 \%$ of the label claim. These studies showed that while the slowdown in dissolution of API 2 was influenced more by the relative humidity than API 1, the overall slowdown in dissolution was more sensitive to changes in temperature than changes in relative humidity. In addition to showing that ASAPprime ${ }^{\circledast}$ could be used to model the effects of temperature and relative humidity on dissolution, the software was also used to demonstrate that no special precautions were necessary to protect the tablets from moisture and they could be stored in Aclar blisters ${ }^{\circledast}$. It was also shown that the water content of the tablet was not a critical quality attribute and need not be included in the drug product specification.
\end{abstract}

Keywords: Accelerated stability, Accelerated stability assessment program (ASAPprime ${ }^{\circledast}$ ), Arrhenius relationship, Dissolution, Stability prediction, Temperature effects, Moisture effects

\footnotetext{
*Correspondence: hanlin_li@vrtx.com

${ }^{1}$ Analytical Development, Vertex Pharmaceuticals, Inc., 50 Northern Avenue,

Boston, MA 02210, USA

Full list of author information is available at the end of the article
} 


\section{Background}

The use of accelerated stability studies is a wellestablished technique that has been widely used for more than 60 years to predict the shelf life and storage conditions of drugs and drug products (Baertschi 2007; Huynh-Ba 2008; Karstensen 1990; Waterman \& Carella 2007). Whereas the experimental approaches may vary between studies, the general principles are the same and are based on the extrapolation of the rate of degradation at two or more temperatures above the intended condition, using the Arrhenius Relationship (Eq. 1).

$$
\ln k=\ln A-\frac{E_{a}}{R T}
$$

where $\mathrm{k}$ is the rate constant for the reaction, $\mathrm{T}$ is the temperature, $\mathrm{A}$ is the collision frequency factor, $\mathrm{E}_{\mathrm{a}}$ is the activation energy and $\mathrm{R}$ is the gas constant. Equation 1 is actually a variation of the empirical relationship developed by van't Hoff in 1887 to describe the effect of temperature on chemical equilibria and used later by Arrhenius who found that the logarithm of the rate (or rate constant) for various reactions was proportional to the reciprocal of the absolute temperature. Predictions of stability based on the Arrhenius relationship (Eq. 1) work well for well-controlled homogenous systems (such as solutions) over relatively small temperature ranges (e.g. $50{ }^{\circ} \mathrm{C}$ ). However, the Arrhenius relationship is less reliable over larger temperature ranges when the dependency of $\mathrm{A}$ and $\mathrm{E}_{\mathrm{a}}$ on $\mathrm{T}$ described by Eyring (Stella, 2000) results in non-linear relationships between $\ln \mathrm{k}$ and $1 / \mathrm{T}$. Another important source of error arises from extrapolation of the data because the confidence interval of the predicted value increases as the difference between the measured and predicted values increases.

Deviations from ideal behavior (Eq. 1) in more complex, heterogenous systems, make the prediction of stability in solid dosage forms (SDF) more difficult. However, Waterman and co-workers (Waterman \& Swanson 2014; MacDonald 2010; Colgan \& Hofer 2015; Stella 2000; Timmerman 2003; Waterman 2011) have shown that the predominant factor that determines the rate of degradation in solid dosage forms, in addition to temperature, is the presence of water. That group has shown the rate of degradation of active pharmaceutical ingredients in tablets and other SDFs may be predicted using a modified Arrhenius equation (Eq. 2), which takes into account the water content as well as the temperature:

$$
\ln k=\ln A-\frac{E_{a}}{R T}+B(R H)
$$

where $\mathrm{B}$ is the humidity sensitivity factor and $\mathrm{RH}$ is the relative humidity. Having demonstrated the applicability of various statistical approaches for the evaluation of

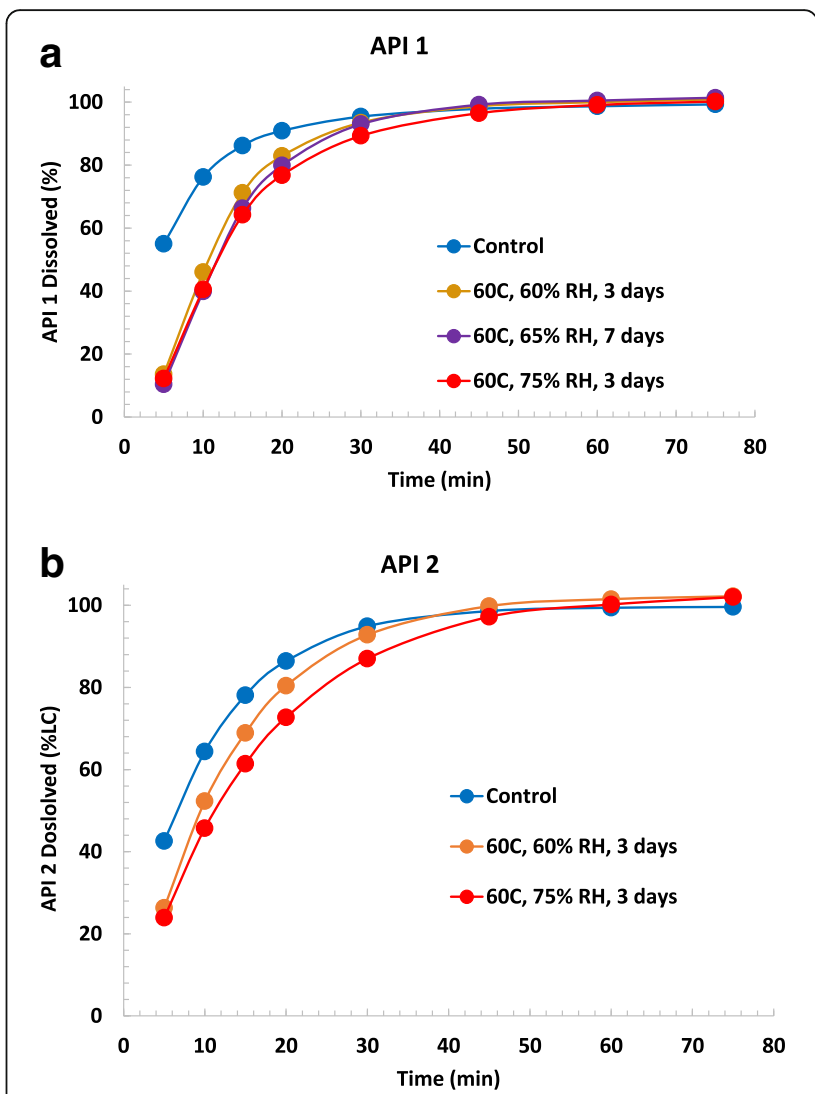

Fig. 1 a Dissolution Profiles of API 1 under Different Storage Conditions, in pH 1.5 Media. b Dissolution Profiles of API 2 under Different Storage Conditions, in pH 6.8 Media

data (Eq. 2) to predict the shelf life of SDFs, Waterman and co-workers (Waterman \& Swanson 2014; MacDonald 2010; Colgan \& Hofer 2015; Stella 2000; Timmerman 2003; Waterman 2011) have developed computer software $(\text { ASAPprime })^{\circ}$ to facilitate the calculations. This approach relies on the measurement of the rates of degradation at

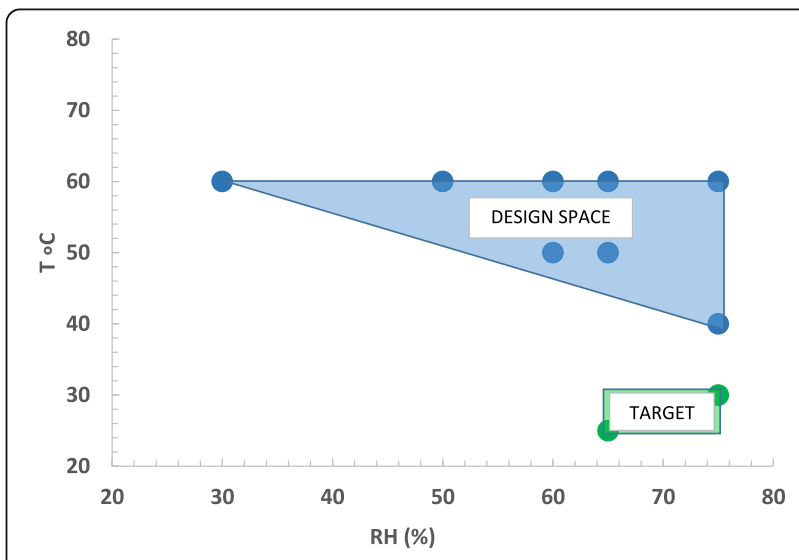

Fig. 2 Design Space showing the Conditions Studied (blue) and the Conditions for the Predictions (green) 
Table $1^{\text {a }}$ Temperature $(\mathrm{T})$ and relative humidity $(\mathrm{RH})$ conditions used in ASAP modeling

\begin{tabular}{llll}
\hline Experiment & $\mathrm{T}\left({ }^{\circ} \mathrm{C}\right)$ & $\mathrm{RH}(\%)$ & Sampling Points (days) \\
\hline 1 & 60 & 30 & $0,7,14$ \\
2 & 60 & 50 & $0,3,7$ \\
3 & 60 & 60 & 0,3 \\
4 & 60 & 65 & $0,3,7$ \\
5 & 60 & 75 & 0,3 \\
6 & 50 & 60 & $0,7,14$ \\
7 & 50 & 65 & $0,7,14$ \\
$8^{\mathrm{b}}$ & 40 & 75 & $0,3,6^{\mathrm{c}}$ months \\
\hline
\end{tabular}

additional stability data at $40{ }^{\circ} \mathrm{C} / 60 \% \mathrm{RH}$ for API 1 from a separate, unrelated study were also included in the modeling for API 1 . The inclusion of the data does not change the overall conclusion for API 1. Data for API 2 at $40{ }^{\circ} \mathrm{C} / 60 \% \mathrm{RH}$ were not available

${ }^{b}$ Open Dish Study

'Only API 1 was tested at 6 months

various temperatures and relative humidities. Equation 2 contains two independent variables ( $\mathrm{T}$ and $\mathrm{RH}$ ); therefore, at least three combinations (a 3-point protocol) of $\mathrm{T}$ and $\mathrm{RH}$ are necessary to obtain values of $\mathrm{A}, \mathrm{E}_{\mathrm{a}}$ and $\mathrm{B}$ by regression analysis or other curve fitting techniques. The greater the number of points in the protocol the greater the reliability of the estimates of the regression coefficients, and the predicted shelf life.

If the stability of the drug product is significantly affected by the presence of moisture, sealing in a waterimpermeable package (such as aluminum foil or blister) may be required to reduce the rate of degradation by eliminating any further ingress of water toward the product. Packaging in a semi-permeable container or blister may slow down the degradation by reducing the moisture vapor transmission rate (MTVR). However, if the product is particularly sensitive to the presence of water, packaging with a desiccant may be necessary. Accelerated stability studies of SDFs are frequently conducted in so called "open dish" studies in which the test article is exposed to the atmosphere and equilibrates with the ambient humidity. An alternative approach to understanding the effects of humidity on the rate of degradation is to first preequilibrate the SDF with an atmosphere of known $\mathrm{RH}$ and then seal it in a water-impermeable pouch made of aluminum foil. In addition to predicting the effects of temperature and relative humidity, ASAPprime ${ }^{\varpi}$ has the added advantage of allowing the effects of packaging in water-impermeable or partially impermeable containers or blisters on stability to be predicted.

Although the intended application of (ASAPprime ${ }^{\oplus}$ ) is the prediction of the rates of chemical degradation in SDFs there is no reason that this approach cannot be applied to physical instability, because Eq. 2 is empirical and simply a way of correlating the rate of change of any measurable parameter of product performance as a function of time, temperature and relative humidity. Accordingly, the objective of this study was to test the hypothesis that ASAPprime ${ }^{ø}$ software can be used to predict the dissolution rate of change of the active ingredients in tablets at the intended storage condition, based on data obtained at elevated temperature and relative humidity. This approach is reasonable because the majority of changes in dissolution rate of SDFs can be attributed to changes in water content (Waterman \& Swanson 2014; MacDonald 2010; Colgan \& Hofer 2015; Stella 2000; Timmerman 2003; Waterman 2011). The SDF used to test the hypothesis was an investigational immediate release tablet containing two active pharmaceutical ingredients (API 1 and API 2) that had previously shown slowed dissolution at $40{ }^{\circ} \mathrm{C} / 75 \% \mathrm{RH}$ in an open dish study and enabled better understanding of the risk to dissolution changes at room-temperature storage.

\section{Results and discussion}

Slowdown in dissolution of both APIs was observed for tablets stored at highly elevated temperature and relative humidity conditions, likely due to physical changes of the SDF, no chemical degradation of either API was observed under these conditions. Representative dissolution profiles are shown in Fig. 1a, which demonstrates that the dissolution of API 1 at $20 \mathrm{~min}$ decreased from an initial value of 90.9 to $82.9 \%$ after three days storage in an open dish at $60{ }^{\circ} \mathrm{C} / 60 \% \mathrm{RH}$. The dissolution of API 1 decreased to $79.9 \%$ after storage in an open dish for 7 days at $60{ }^{\circ} \mathrm{C} /$ $65 \% \mathrm{RH}$ and $76.8 \%$ after 3 days at $60{ }^{\circ} \mathrm{C} / 75 \% \mathrm{RH}$. A similar slowdown in the dissolution of API 2 was also observed (Fig. 1b).

The impact of temperature and RH (30-75\%) (T/RH) on dissolution at long term storage conditions was evaluated through a number of stressed and open dish studies. The dissolution changes at $20 \mathrm{~min}$ for both APIs over long term storage are of particular interest,

Table 2 Stability studies/conditions used for external model validation

\begin{tabular}{llllll}
\hline Study & $\mathrm{T}\left({ }^{\circ} \mathrm{C}\right)$ & $\mathrm{RH}(\%)$ & Time Points for Validation & Packaging Configurations & Initial Tablet Water Content \\
\hline 1 & 25 & 60 & 12 months & Aclar Blisters & \\
& 30 & 65 & 12 months & & \\
& 40 & 75 & 6,12 months & & $4.1 \%$ \\
\hline
\end{tabular}


Table 3 Regression coefficients for data fitted to the modified Arrhenius equation (Eqs. 2 and 3) and the Dimensionless equation (Eq. 4) for the two active ingredients

\begin{tabular}{llllll}
\hline API & $\ln A$ & $E_{a}(\mathrm{kcal} / \mathrm{mol})$ & $C\left(\times 10^{-4}\right)$ & $\mathrm{B}\left(\times 10^{-2}\right)$ & $\mathrm{R}^{2}$ \\
& $(\mathrm{SD})$ & $(\mathrm{SD})$ & $\left(\mathrm{SD} \times 10^{-4}\right)$ & $\left(\mathrm{SD} \times 10^{-2}\right)$ & \\
\hline 1 & $73.8(5.7)$ & $50.0(3.9)$ & $2.52(0.02)$ & $5.3(0.8)$ & 0.937 \\
2 & $71.4(9.7)$ & $51.0(6.6)$ & $2.58(0.03)$ & $10.4(1.9)$ & 0.954 \\
\hline
\end{tabular}

as typical dissolution specifications for immediate release tablets are $\mathrm{Q}=80 \%$ at $20 \mathrm{~min}$. (The quantity, $\mathrm{Q}$, as defined per USP $<711>$, is the amount of dissolved active ingredient, in percent label claim). The data were analyzed by ASAPprime ${ }^{\oplus}$ to develop a model describing the effect of moisture and temperature on dissolution over the product shelf life at target storage conditions. The T/RH design space and target are shown in Fig. 2. Detailed experimental design and durations are shown in Table $1 .^{1}$ The predicated data from ASAPprime $e^{\curvearrowleft}$ modeling were compared against real-time stability data to validate the approach (Table 2). The experimental data used to construct the model relating the percent dissolved to values of $\mathrm{T}$ and $\mathrm{RH}$ were generated by pre-equilibrating the tablets at $25{ }^{\circ} \mathrm{C}$ and various values of $\mathrm{RH}(30-75 \%)$ (Fig. 2). The mean values for the amounts of each API dissolved $(n=3)$ after $20 \mathrm{~min}$ are provided in Appendix 1. The data were fitted to Eq. 2 and the value for the regression coefficients

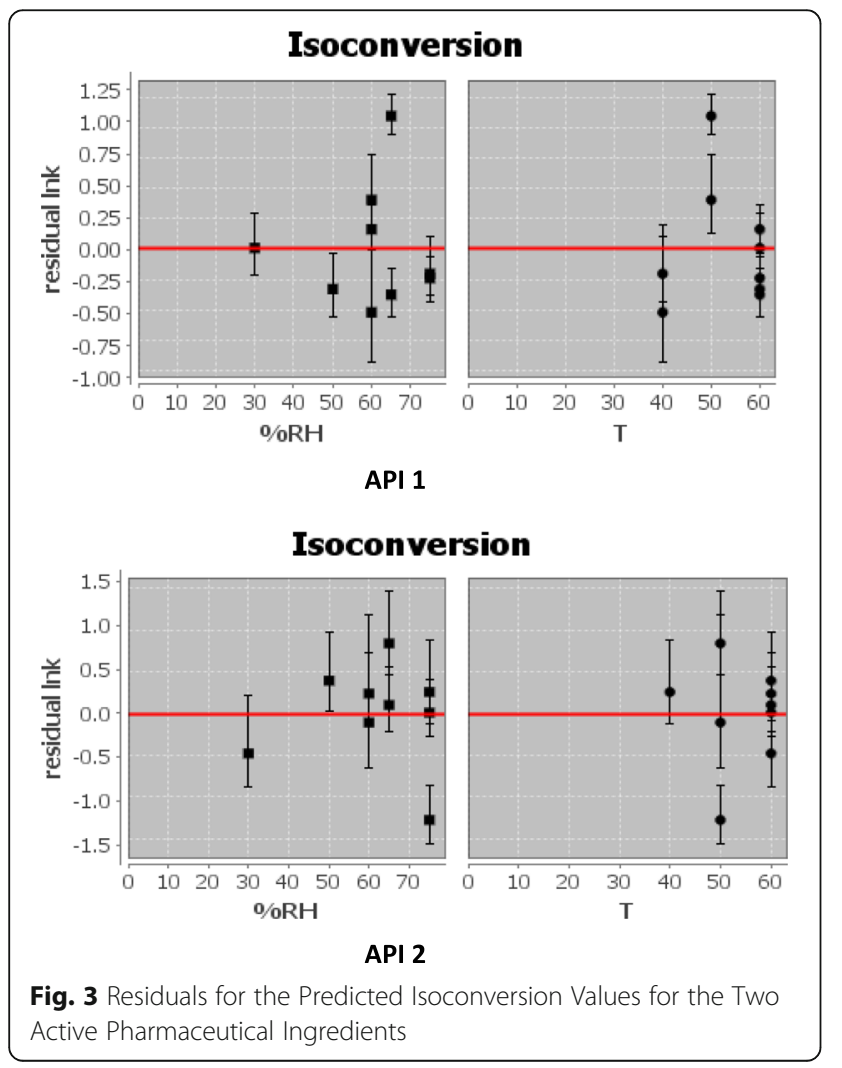

(A, $E_{a}, B$ and $R^{2}$ ) are shown in Table 3 . The values of 0.937 and 0.954 for $\mathrm{R}^{2}$ mean that $6.3 \%$ (API 1 ) and $4.6 \%$ (API 2) of the variance in the data for the two APIs are not explained by the model. Figure 3 shows the residuals obtained from the predicted values and those obtained by experiment were randomly distributed, indicating that there was no systematic error in the predictions.

\section{Effects of temperature and relative humidity on dissolution}

The computer software (ASAPprime ${ }^{\circ}$ ) uses the isoconversion method (Waterman (2011)), rather than regression analysis to calculate the values of $\mathrm{A}, \mathrm{E}_{\mathrm{a}}, \mathrm{B}$, where the isoconversion time, IC is the time to reach the predetermined value for the acceptance criteria (not less $80 \%$ dissolved after $20 \mathrm{~min}$ ). Replacement of the rate constant, $\mathrm{k}$ with the reciprocal of IC gives:

$$
\ln \frac{1}{I C}=\ln A-\frac{E_{a}}{R T}+B(R H)
$$

Equation 3 can be re-written in dimensionless terms by substitution of $\frac{E_{a}}{R}$ by $\mathrm{C}$,

$$
\ln \frac{1}{I C}=\ln A-\frac{C}{T}+B(R H)
$$

where $\mathrm{C}$ is the temperature sensitivity factor. Equation 4 allows the effects of changing $\mathrm{T}$ and $\mathrm{RH}$ to be assessed independently of each other. The effect of changing the atmospheric moisture from a value of $\mathrm{RH}_{1}$ to $\mathrm{RH}_{2}$ at a fixed value of $\mathrm{T}(\Delta R H)$ may be determined as follows:

$$
\ln \frac{I C_{R H 2}}{I C_{R H 1}}=B\left(R H_{1}-R H_{2}\right)
$$

Equation 5 can be re-written in non-logarithmic terms to give:

$$
\frac{I C_{R H 2}}{I C_{R H 1}}=F_{\Delta R H}=e^{B\left(R H_{1}-R H_{2}\right)}
$$

where $F_{\triangle R H}$ is the factor by which the isoconversion time (the time to reach $\mathrm{Q}=80 \%$ ) changes for a given change in $\mathrm{RH}(\Delta \mathrm{RH})$. Equation 6 predicts that the value of $F_{\Delta R H}$ is independent of the intital and final values of $\mathrm{RH}$ and depends only on the difference. Therefore, the values of $F_{\Delta R H}$, for $10 \%$ change in absolute $\mathrm{RH}(\Delta \mathrm{RH}=10 \% \mathrm{RH})$ are 1.70 and 2.83 for API 1 and API 2, respectively. This means that the change in isoconversion time for the two APIs for a $10 \%$ change in absolute $\mathrm{RH}$ is approximately $70 \%$ for API 1 and approximately $183 \%$ for API 2 at a fixed temperature.

The effect of changing $\mathrm{T}$ at a fixed value of $\mathrm{RH}(\Delta T)$ is slightly more complicated because the value of $F_{\Delta T}$, depends on the initial, $\left(T_{1}\right)$ and the final temperature $\left(T_{2}\right)$, as follows: 

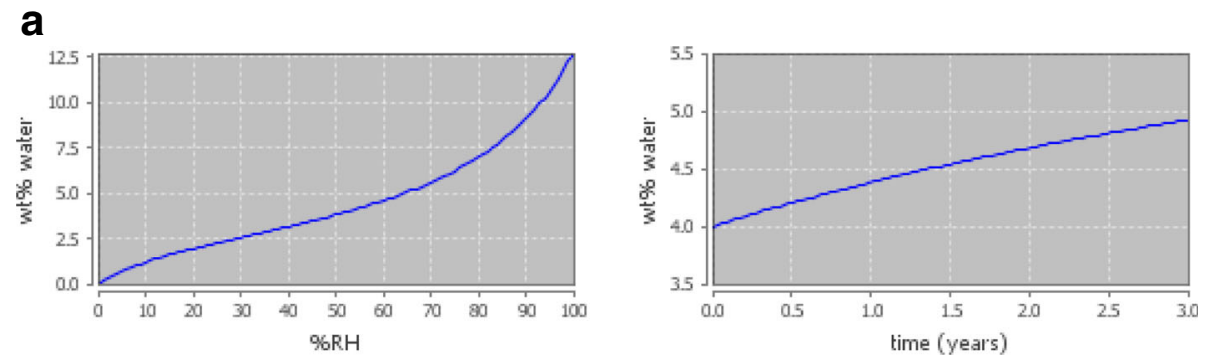

\section{b Predicted \% Water at 25C/60\%RH}

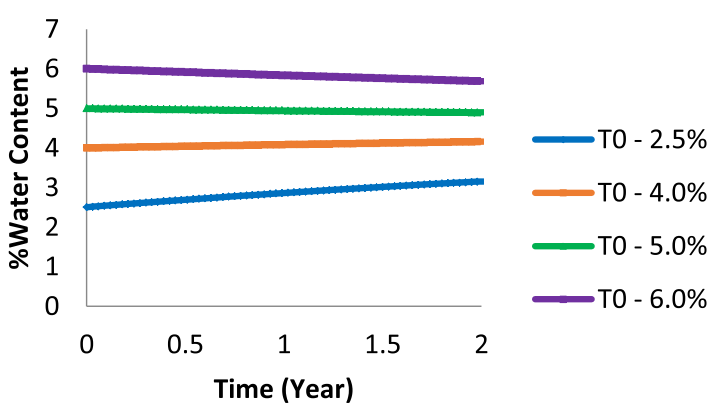

C Predicted \%water at $30 \circ \mathrm{C} / 75 \% \mathrm{RH}$

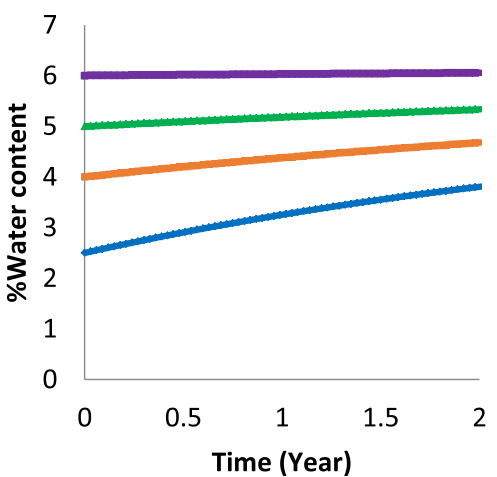

Fig. 4 a Predicted Water Isotherm (left) and the Predicted Water Content in the Tablets after Open-Dish Storage at $25^{\circ} \mathrm{C} / 60 \% \mathrm{RH}$ (initial water content $4.0 \%$ ). b Predicted Water Contents in Tablets Packaged in Aclar Blisters Over 2 Years at $25^{\circ} \mathrm{C} / 60 \% \mathrm{RH}$ with Different Initial Water Contents. c Predicted Water Contents in Tablets Packaged in Aclar Blisters Over 2 Years at $30^{\circ} \mathrm{C} / 75 \% \mathrm{RH}$ with Different Initial Water Contents

$$
\ln \frac{I C_{T 2}}{I C_{T 1}}=C\left(\frac{1}{T_{2}}-\frac{1}{T_{1}}\right)
$$

or

$$
\ln \frac{I C_{T 2}}{I C_{T 1}}=C\left(\frac{T_{1-} T_{2}}{T_{1} T_{2}}\right)
$$

and

$$
\frac{I C_{T 2}}{I C_{T 1}}=F_{\Delta T}=e^{C\left(\frac{T_{1}-T_{2}}{T_{1} T_{2}}\right)}
$$

That being said, the error in using a constant value of the product $\mathrm{T}_{1} \mathrm{~T}_{2}$ is less than $10 \%$ over relatively small temperature ranges $\left(20-60{ }^{\circ} \mathrm{C}\right)$, in which case Eq. 9 simplifies to:

$$
\frac{I C_{T 2}}{I C_{T 1}}=F_{\Delta T}=e^{C \Delta \mathrm{T} \times 10^{-4}}
$$

Therefore, the values of $F_{\Delta T}$, for $10{ }^{\circ} \mathrm{C}$ change in temperature are 12.4 and 11.0 for API 1 and API 2, respectively. This means that the change in isoconversion time for a $10{ }^{\circ} \mathrm{C}$ change in temperature for API 1 and API 2 are approximately $1250 \%$ and $1100 \%$, respectively, at a fixed value of $\mathrm{RH}$.

It can be shown that the combined effect changing both $\mathrm{T}$ and $\mathrm{RH}\left(F_{\Delta T, \Delta R H}\right)$ can be estimated by: 


$$
F_{\Delta T, R H}=e^{\left(B \Delta R H+C \Delta \mathrm{T} \times 10^{-4}\right)}
$$

or

$$
\begin{aligned}
F_{\Delta T, R H} & =F_{\Delta T} \quad \times \quad F_{\Delta R H} \\
& =\left(e^{B \Delta R H}\right) \times e^{\left(C \Delta \mathrm{T} \times 10^{-4}\right)}
\end{aligned}
$$

Therefore, the combined effect of changing the storage conditions from the accelerated conditions $40{ }^{\circ} \mathrm{C} / 75 \%$ $\mathrm{RH}$ to the intended storage condition of $25^{\circ} \mathrm{C} / 60 \% \mathrm{RH}$ $\left(\Delta \mathrm{T}=15{ }^{\circ} \mathrm{C} \Delta \mathrm{RH}=15 \% \mathrm{RH}\right)$ predicted by the model $\left(F_{\Delta T} \times F_{R H}\right)$ is 96.5 and 181.3, for API 1 and API 2, respectively. The individual contributions from temperature and relative humidity are as follows

API 1

$$
\begin{aligned}
& F_{\Delta T}=43.6 \\
& F_{R H}=2.21
\end{aligned}
$$

API 2

$$
\begin{aligned}
& F_{\Delta T}=38.9 \\
& F_{R H}=4.66
\end{aligned}
$$

It is clear from Eqs. 13-16 that the contribution to a reduction in dissolution at $20 \mathrm{~min}$ for both drugs from elevating the temperature is significantly greater than the contribution from increasing the relative humidity and that the effects of temperature on the dissolution of both drugs is about the same. Interestingly, although the effects of humidity change were relatively small, the effect of RH on the dissolution of API $1\left(F_{R H}=2.21\right)$ was much less than the effect on API $2\left(F_{R H}=4.66\right)$. The reason for the difference in the effect of water on the dissolution of the two APIs is not clear; however, it does have important implications for the stability of the tablets at room temperature, which are discussed in the next sections.

\section{Effects of initial water content}

The weak dependency of drug dissolution on RH (Eq. 4) was investigated further to determine if it was necessary to control the water content of the tablets in the drugproduct specification. The amount of water taken up by the tablets at specific values of $\mathrm{RH}$ was entered into ASAPprime $^{\oplus}$ to determine the water sorption isotherm over the entire range of $\mathrm{RH}$ (Fig. $4 \mathrm{a}) .^{2}$ Fig. $4 \mathrm{a}$ shows a sigmoidal relationship between the water content of the tablets in which the water content increases slowly up to $50 \% \mathrm{RH}$ to a value of $4 \%$ and then increases exponentially to approximately $13 \%$ at $100 \% \mathrm{RH}$. Tablet water content change on stability can also be predicted based on the GAB parameters and given packaging configurations. Figure $4 \mathrm{~b}$ and $\mathrm{c}$ are examples of predicted tablet water contents under $25{ }^{\circ} \mathrm{C} / 60 \% \mathrm{RH}$ and $30{ }^{\circ} \mathrm{C} / 75 \% \mathrm{RH}$ storage conditions over 2 years at different initial values. The software uses this information to predict the effect of the water content on the dissolution of the tablets. Figure 5 shows the anticipated effect that the amount of drug dissolved at 20 mins after two years' storage decreases and the effect is more pronounced for API 1 than for API 2, which is expected from the previous discussions described above. Although the effect of humidity is much less for API 1 than API 2, the dissolution rate drop for both APIs are primarily driven by effect of temperature, which is more significant for API 1 than API 2; therefore, more changes are observed in API 1 than API 2 at the end of the two-year storage and the differences are more pronounced at $30{ }^{\circ} \mathrm{C}$ than at $25{ }^{\circ} \mathrm{C}$. Despite the differences in the effects of initial moisture on the dissolution of the two APIs, ASAPprime ${ }^{\bullet}$ predicts that the dissolution of both drugs will remain within specification after storage $(\mathrm{Q}=80 \%)$ for two years at either $25{ }^{\circ} \mathrm{C} / 60 \% \mathrm{RH}$, or $30{ }^{\circ} \mathrm{C} / 75 \% \mathrm{RH}$ independent of the initial water content up to $6.3 \%$ water, which is the equilibrium water content of the tablets at $75 \% \mathrm{RH}$. The equilibrium water content at $60 \% \mathrm{RH}$ was found to be $4.1 \%$.

\section{Stability in Aclar $^{\circledast}$ blisters}

Although the ASAPprime $e^{\odot}$ analysis predicted some differences in the sensitivity of the dissolution of the two APIs to moisture, the results also indicated the effect of $\mathrm{RH}$ was insufficient to warrant the need for special packaging. This is supported by the results of two studies summarized in Table 4, which show very little, if any, increase in moisture content of the tablets packaged in Aclar blisters for three to six months at various storage conditions. Table 4 also shows very good agreement between the observed values of water content and the

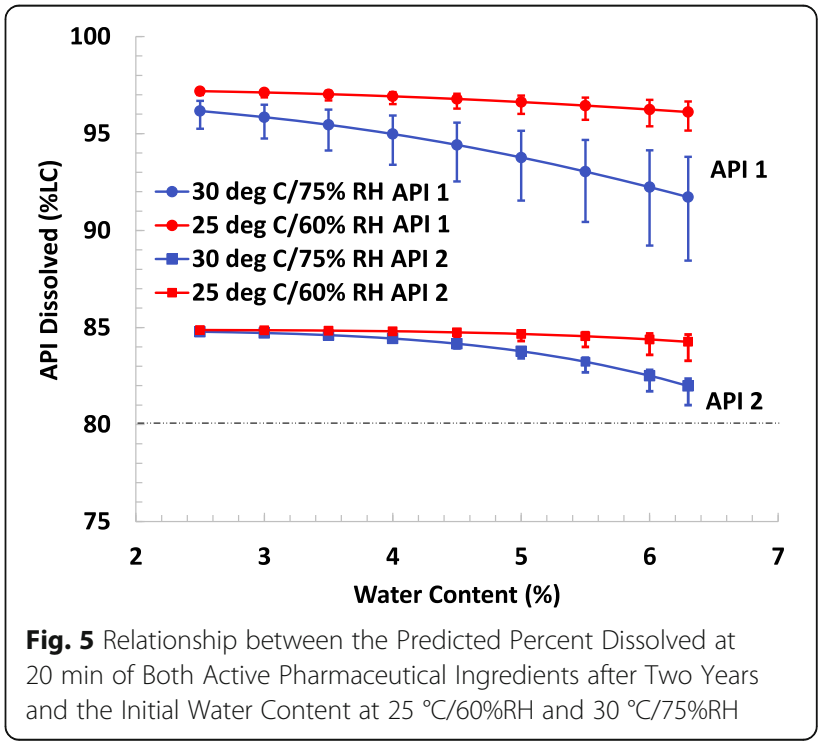


Table 4 Predicted and observed water content of tablets packaged in Aclar ${ }^{\oplus}$ foil blisters at different storage conditions (Temperature $(\mathrm{T})$ and Relative Humidity $(\mathrm{RH})$ )

\begin{tabular}{|c|c|c|c|c|c|c|}
\hline \multirow[t]{2}{*}{ Study } & \multicolumn{3}{|c|}{ Storage Conditions } & \multicolumn{3}{|c|}{ Water Content (\% w/w) } \\
\hline & $\mathrm{T}\left({ }^{\circ} \mathrm{C}\right)$ & $\mathrm{RH}(\%)$ & Time (months) & Initial & Predicted & Observed \\
\hline \multirow[t]{3}{*}{1} & 30 & 75 & 6 & 2.1 & 2.4 & 2.2 \\
\hline & 40 & 75 & 6 & & 2.8 & 2.7 \\
\hline & 25 & 60 & 6 & & 2.3 & 2.0 \\
\hline \multirow[t]{3}{*}{2} & 25 & 60 & 3 & 2.4 & 2.5 & 2.4 \\
\hline & 30 & 65 & 3 & & 2.5 & 2.3 \\
\hline & 40 & 75 & 3 & & 2.8 & 2.7 \\
\hline
\end{tabular}

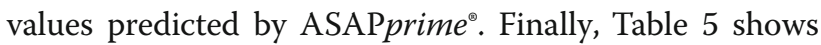
no difference in the amounts of the two APIs dissolved from tablets, packaged in Aclar ${ }^{\oplus}$ blisters, following storage for 12 months at $25{ }^{\circ} \mathrm{C} / 60 \% \mathrm{RH}, 30{ }^{\circ} \mathrm{C} / 65 \% \mathrm{RH}$ and $40{ }^{\circ} \mathrm{C} / 75 \% \mathrm{RH}$. Similar results were observed on a different batch of tablets stored at the same conditions

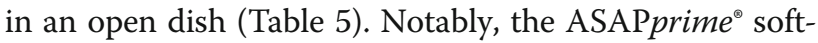
ware predicted very accurately that the change in dissolution after storage of the tablets packaged in Aclar ${ }^{\circ}$ blisters would be insignificant after storage for 12 months at the intended storage condition $\left(25^{\circ} \mathrm{C} / 60 \% \mathrm{RH}\right)$. We will continue to the monitor the stability of these formulations with a view to establishing a two-year shelf life, a period over which predictions made by the ASAPprime software indicated that the dissolution of the two drugs would be well within typical specifications $(\mathrm{Q}=80 \%$ at $20 \mathrm{~min}$ ) for an immediate release product.

\section{Conclusions}

In this study, we have clearly demonstrated that the computer program, ASAPprime ${ }^{\ominus}$, originally developed to predict the rates of degradation of SDFs arising from chemical reactions can also be used to predict changes in dissolution. Experiments conducted under various conditions of elevated temperature and relative humidity accurately predicted that the reduced rates of dissolution of two APIs in an SDF, IR tablet seen under accelerated conditions would not translate into any measurable changes in dissolution over the shelf life of the product (up to two years at $25{ }^{\circ} \mathrm{C} / 60 \% \mathrm{RH}$ or $30{ }^{\circ} \mathrm{C} / 75 \% \mathrm{RH}$ ). Furthermore, the software predicted accurately that no special precautions are necessary to protect the product from moisture and that there is no need to control the water content of the tablets in the drug product specification. The methodology described here adds great value to the overall Quality by Design approach to tablet development.

\section{Methods \\ Materials \\ Chemicals and reagents}

All chemicals and reagents, obtained from various commercial sources, were reagent grade, except for the HPLC solvents, which were HPLC grade. Deionized water was prepared in-house.

\section{Tablets}

The article used to test the hypothesis was an investigational, solid dosage form (SDF) tablet ${ }^{3}$ containing two active pharmaceutical ingredients, API 1 and API 2: both drugs were small molecules with molecular weights of less than 1000 .

\section{Procedures}

\section{Dissolution procedure}

Tablet dissolution testing was performed on three tablets stored at each condition using USP Apparatus 2 in $900 \mathrm{~mL}$ of dissolution media at $37{ }^{\circ} \mathrm{C}$. Due to their

Table 5 Predicted and observed dissolution of the two active pharmaceutical ingredients after 20 min at different storage conditions of temperature $(\mathrm{T})$ and relative humidity $(\mathrm{RH})$

\begin{tabular}{|c|c|c|c|c|c|c|c|c|c|}
\hline \multirow[t]{4}{*}{ Study } & \multicolumn{3}{|c|}{ Storage Conditions } & \multicolumn{6}{|c|}{ Amount Dissolved (LC\%) } \\
\hline & \multirow[t]{3}{*}{$\mathrm{T}\left({ }^{\circ} \mathrm{C}\right)$} & \multirow[t]{3}{*}{$\mathrm{RH}(\%)$} & \multirow[t]{3}{*}{ Time (months) } & \multicolumn{3}{|l|}{$\overline{\mathrm{API}} 1$} & \multicolumn{3}{|l|}{ API 2} \\
\hline & & & & \multirow[t]{2}{*}{ Initial (T0) } & \multicolumn{2}{|c|}{ Final (6 or 12 mo) } & \multirow[t]{2}{*}{ Initial } & \multicolumn{2}{|c|}{ Final (6 or $12 \mathrm{mo}$ ) } \\
\hline & & & & & Predicted & Observed & & Predicted & Observed \\
\hline \multirow[t]{4}{*}{$1^{a}$} & 25 & 60 & 12 & \multirow[t]{4}{*}{95.9} & 95.9 & 95.7 & \multirow[t]{4}{*}{85.5} & 84.9 & 87.4 \\
\hline & 30 & 65 & 12 & & 95.8 & 96.4 & & 84.8 & 86.7 \\
\hline & 40 & 75 & 6 & & 94.7 & 92.5 & & 84.6 & 85.6 \\
\hline & 40 & 75 & 12 & & 92.7 & 92.5 & & 83.9 & 84.8 \\
\hline \multirow[t]{2}{*}{$2^{b}$} & 25 & 60 & 6 & \multirow[t]{2}{*}{92.9} & 92.8 & 94.1 & \multirow[t]{2}{*}{86.3} & $N T^{c}$ & $N T^{c}$ \\
\hline & 25 & 60 & 12 & & 92.7 & 93.2 & & 84.8 & 87.1 \\
\hline
\end{tabular}

${ }^{a}$ Tablets packaged in Aclar ${ }^{\circledast}$ blisters. Initial water content $2.1 \%$

${ }^{b}$ Open dish study. Initial water content $4.1 \%$

${ }^{\mathrm{c}}$ Not tested 
different aqueous solubilities, the dissolution profile of API 1 was obtained with $\mathrm{pH} 1.5$ media, and the dissolution profile of API 2 was obtained with $\mathrm{pH} 6.8$ media. Dissolution profiles were collected at 5, 10, 15, 20, 30, 45 and $60 \mathrm{~min}$ at $75 \mathrm{rpm}$ and $65 \mathrm{rpm}$, respectively for API 1 and API 2, with an "infinity spin" from 60-75 min at $200 \mathrm{rpm}$.

\section{Sample analysis}

Samples $(1.5 \mathrm{~mL})$ were removed and filtered immediately through a polyvinylidene fluoride (PVDF, $10 \mu \mathrm{m}$,) filter at the sampling times listed above and analyzed for API content by HPLC. The HPLC procedure was validated for accuracy, precision and linearity and found to be suitable for the intended application. The system was calibrated using external standards containing concentrations of API 1 and API 2 equivalent to $100 \%$ of the label claims (LC) of each API.

\section{Stability studies}

In one set of experiments the tablets were preequilibrated to constant water content at various relative humidity $(\mathrm{RH})$ values ranging from $30 \%$ to $75 \%$ at $25{ }^{\circ} \mathrm{C}$ sealed in aluminum foil pouches and stored for various periods of time (Table 2) at $50{ }^{\circ} \mathrm{C}$ and $60{ }^{\circ} \mathrm{C}$. In a second set of experiments tablets were stored in petri dishes (open dish) at $40{ }^{\circ} \mathrm{C} / 75 \% \mathrm{RH}$. Detailed experimental conditions and durations are listed in Table 1. The samples were stored in calibrated stability chambers.

\section{Water content}

The water content of the tablets was determined by automated Karl Fischer titration.

\section{Water sorption isotherm}

The water sorption isotherm of the tablets was determined using a dynamic water sorption analyzer.

\section{Data analysis}

The concentrations of the dissolved APIs at each sampling point $\left(\mathrm{C}_{1, \mathrm{t}}\right.$ and $\left.\mathrm{C}_{2, \mathrm{t}}\right)$ were expressed as a percentage of the label claim $\left(\mathrm{LC} \%_{1, \mathrm{t}}\right)$ (Eq. 17) and plotted against time, $\mathrm{t}$ to generate the dissolution profiles $\left(\mathrm{LC}_{1 \text { (or } 2), \mathrm{t}} \mathrm{vs} . \mathrm{t}\right)$.

$$
L C \%_{(1 \text { or } 2), t}=\frac{C_{1(\text { or } 2), t}}{L C_{1(\text { or } 2), t}} 100 \%
$$

The relationships between the values of $\mathrm{LC} \%_{1,20}$ and $\mathrm{LC} \%_{2,20}$, temperature $(\mathrm{T})$ and relative humidity $(\mathrm{RH})$ were established by fitting the data Eq. 2 to calculate the regression coefficients, $\mathrm{A}, \mathrm{E}_{\mathrm{a}}$, and $\mathrm{B}$, using the ASAPprime $^{\varpi}$ software (version 3, FreeThink Technologies, Inc. Montvale, NJ 06405).

\section{Endnotes}

${ }^{1}$ Additional stability data at $40{ }^{\circ} \mathrm{C} / 60 \% \mathrm{RH}$ for API 1 from a separate, unrelated study were also included in the modeling for API 1. The inclusion of the data does not change the overall conclusion for API 1. Data for API 2 at $40{ }^{\circ} \mathrm{C} / 60 \% \mathrm{RH}$ were not available.

${ }^{2}$ The software uses the GAB equation developed by Guggenheim, Anderson and de Boer as described by Timmerman (Waterman 2009) to simulate the water sorption isotherm. The GAB Equation parameters are provided in Appendix 2.

${ }^{3}$ The structures of the APIs and the composition of the tablets are proprietary; however, disclosure of this information is not necessary to understand how the study was conducted, the hypothesis tested or the conclusions.

\section{Appendix 1}

Table 6 ASAP data input for API 1 - dissolution at 20 min

\begin{tabular}{|c|c|c|c|c|}
\hline Time (days) & $\mathrm{T}\left({ }^{\circ} \mathrm{C}\right)$ & $\% \mathrm{RH}$ & $\%$ LC at $20 \mathrm{~min}$ & SD (\%) \\
\hline 0 & 60 & 60 & 98.1 & 0.9 \\
\hline 3 & 60 & 60 & 82.9 & 3 \\
\hline 0 & 60 & 75 & 96.2 & 0.9 \\
\hline 3 & 60 & 75 & 76.8 & 3 \\
\hline 0 & 40 & 75 & 96.3 & 0.9 \\
\hline 90 & 40 & 75 & 90.9 & 3 \\
\hline 180 & 40 & 75 & 86.7 & 3 \\
\hline 0 & 60 & 30 & 98.4 & 0.9 \\
\hline 7 & 60 & 30 & 90 & 3 \\
\hline 14 & 60 & 30 & 85.6 & 3 \\
\hline 0 & 60 & 50 & 98.1 & 0.9 \\
\hline 3 & 60 & 50 & 87.6 & 3 \\
\hline 7 & 60 & 50 & 85.8 & 3 \\
\hline 0 & 60 & 65 & 97.5 & 0.9 \\
\hline 3 & 60 & 65 & 79.9 & 3 \\
\hline 7 & 60 & 65 & 76.4 & 3 \\
\hline 0 & 50 & 60 & 98.1 & 0.9 \\
\hline 7 & 50 & 60 & 92.4 & 3 \\
\hline 14 & 50 & 60 & 88.9 & 3 \\
\hline 0 & 50 & 65 & 97.5 & 0.9 \\
\hline 7 & 50 & 65 & 87 & 3 \\
\hline 14 & 50 & 65 & 76.4 & 3 \\
\hline 0 & 40 & 60 & 96.2 & 0.9 \\
\hline 90 & 40 & 60 & 94.8 & 3 \\
\hline 180 & 40 & 60 & 93.7 & 3 \\
\hline
\end{tabular}


Table 7 ASAP data input for API 2 - dissolution at 20 min

\begin{tabular}{|c|c|c|c|c|}
\hline Time (days) & $\mathrm{T}\left({ }^{\circ} \mathrm{C}\right)$ & $\% \mathrm{RH}$ & $\%$ LC at $20 \mathrm{~min}$ & SD (\%) \\
\hline 0 & 60 & 60 & 84.9 & 1.1 \\
\hline 3 & 60 & 60 & 77.3 & 3 \\
\hline 0 & 60 & 75 & 84.7 & 1.1 \\
\hline 3 & 60 & 75 & 70.5 & 3 \\
\hline 0 & 40 & 75 & 86.3 & 1.1 \\
\hline 90 & 40 & 75 & 80.6 & 3 \\
\hline 0 & 60 & 30 & 85.5 & 1.1 \\
\hline 7 & 60 & 30 & 85.5 & 3 \\
\hline 14 & 60 & 30 & 84.2 & 3 \\
\hline 0 & 60 & 50 & 85.9 & 1.1 \\
\hline 3 & 60 & 50 & 80 & 3 \\
\hline 7 & 60 & 50 & 79.3 & 3 \\
\hline 0 & 60 & 65 & 83.4 & 1.1 \\
\hline 3 & 60 & 65 & 76 & 3 \\
\hline 7 & 60 & 65 & 76.3 & 3 \\
\hline 0 & 50 & 60 & 84.9 & 1.1 \\
\hline 7 & 50 & 60 & 83.8 & 3 \\
\hline 14 & 50 & 60 & 82.7 & 3 \\
\hline 0 & 50 & 65 & 83.4 & 1.1 \\
\hline 7 & 50 & 65 & 80.8 & 3 \\
\hline 14 & 50 & 65 & 80.8 & 3 \\
\hline
\end{tabular}

\section{Appendix 2}

Table 8 GAB parameters used based on DVS of SDF tablets

\begin{tabular}{lll}
\hline$W_{m}$ & $C$ & $K$ \\
\hline 2.758 & 7.67 & 0.785
\end{tabular}

\section{Authors' contributions}

HL conducted the experimental work. DN was involved in the study's conception and design. $\mathrm{HL}$ and CMR analyzed the data and prepared the abstract, introduction, experimental, results and discussion and conclusions sections. DN and AK reviewed and edited the manuscript. All authors have read and approved the final manuscript.

\section{Competing interests}

Christopher M. Riley, Ph.D. Editor-in-Chief, AAPS Open, was not involved in the review process of this article or in the decision to publish.

\section{Author details}

${ }^{1}$ Analytical Development, Vertex Pharmaceuticals, Inc., 50 Northern Avenue, Boston, MA 02210, USA. ${ }^{2}$ Riley and Rabel Consulting Services, Inc., 1055 Forest Street, Maryville, MO 64468, USA.

Received: 15 June 2016 Accepted: 21 October 2016

Published online: 03 November 2016

\section{References}

Baertschi S (2007) Pharmaceutical Stress Testing: Predicting Drug Degradation. Informa Healthcare, New York

Colgan S, Hofer R, Vuoviski K, Waterman K, Norris (2015) Lean Stability, AAPS Newsmagazine, 14-18 Sept. The Timmerman. vol 220 (1-3)

Huynh-Ba K (2008) Handbook of Stability Testing on Pharmaceutical Development: Regulations, Methodologies and Best Practices. Spirnger, New York

Karstensen J (1990) Drug Stability: Principles and Practices. Marcel Dekker, New York MacDonald KW (2010) Package selection for moisture protection for solid, oral drug products. J Pharm Sci 99:4437-4452

Stella SY (2000) Stability of Drugs and Dosdage Forms. Kluwer Academic/Plenum, New York

Timmerman E (2003) Multilayer sorption parameters: BET or GAB values. Colloid Surf A: Physiochem Eng Asp: 220(1-3):235-360

Waterman K (2009) Understanding and Predicting Pharmaceutical Proruct ShelfLife. In: Huynh-Ba K (ed) Handbook of Stability Testing in Pharmaceutical Development: Regulations, Methodlogies and Best Practices. Springer, New York, pp 115-135

Waterman K (2011) The application of accelerated stability assessment program (ASAP) to quakity by design (QbD) for drug product stability. AAPS PharmSciTech 12(3):932-937

Waterman KC, Carella AJ (2007) Improved protocol and data analysis for accelerated shelf-life estimation of solid dosage forms. Pharm Res 24:780-790

Waterman KC, Swanson JT (2014) A scientific and statisitical analysis of accelerated aging of pharmaceuticals. Part 1: accuracy of fitting methods. J Pharm Sci 103:3000-3006

\section{Abbreviations}

A: Collision frequency factor; API: Active pharmaceutical ingredient; ASAP: Accelerated stability assessment program; B: Humidity sensitivity factor: $C$ : Temperature sensitivity factor; $E_{\mathrm{a}}$ : Activation energy; $F_{\triangle R H}$ : Factor by which the $\mathrm{IC}$ changes for given change in $\mathrm{RH}$ at fixed $\mathrm{T}_{;} F_{\Delta T}$ : Factor by which the $\mathrm{IC}$ changes for given change in T at fixed value of RH; HPLC: High performance liquid chromatography; IC: Isoconversion time; IR: Immediate release; LC: Label claim; LC\%: Percentage label claim; MVTR: Moisture vapor transmission rate; Q: USP acceptance criteria; R: Gas constant; RH: Relative humidity; SDF: Solid dosage form; T: Temperature

\section{Acknowledgements}

The authors gratefully acknowledge the technical contributions of Laurent Bagnol, Ricardo Borjas, Rajesh Penumatcha, Chris Rego, Hailey McDonalds, Alex Scangas, Ales Medek, Hong-Ren Wang and Gerry Enos, Vertex Pharmaceuticals, Inc.

\section{Declarations}

Dissolution predictions and results described herein do not relate in any way to unexpected effects of combining two APIs on the dissolution rate of each individual API.

\section{Submit your manuscript to a SpringerOpen ${ }^{\circ}$ journal and benefit from:}

- Convenient online submission

- Rigorous peer review

- Immediate publication on acceptance

- Open access: articles freely available online

- High visibility within the field

- Retaining the copyright to your article

Submit your next manuscript at springeropen.com 